\title{
Axial Vector Duality in Affine NA Toda Models
}

\author{
J.F. Gomes G.M. Sotkov* and A.H. Zimerman \\ Instituto de Física Teórica - IFT/UNESP, \\ Rua Pamplona 145 \\ 01405-900, São Paulo - SP, Brazil \\ E-mail sotkoveift.unesp. bri
}

Abstract: A general and systematic construction of Non Abelian affine Toda models and its symmetries is proposed in terms of its underlying Lie algebraic structure. It is also shown that such class of two dimensional integrable models naturally leads to the construction of a pair of actions related by T-duality transformations.

\section{Introduction}

Two dimensional integrable models represent an important laboratory for testing new ideas and developing new methods for constructing exact solutions as well as for the nonperturbative quantization of 4-D non-abelian gauge theories, gravity and string theory. Among the numerous techniques for constructing 2-D integrable models and their solutions [i.1], [2i], the hamiltonian reduction of the Wess-Zumino-Witten (WZW) model (or equivalently the gauged WZW ) associated to a finite dimensional Lie algebra $\mathcal{G}$ has provided an universal and simple method for deriving the equations of motion (or action ) of 2-d integrable models. In particular, the conformal Toda $(\mathrm{CT})$ models were constructed by implementing a consistent set of constraints on the WZW currents [3] [3]. The method was subsequently extended to construct the conformal affine Toda models (CAT) from infinite dimensional affine algebras, leading to WZW currents satisfying the so called two loop current algebra

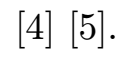

The affine Toda models consists of a class of relativistic two dimensional integrable models admiting soliton solutions with non trivial topological charge (e.g. the abelian affine Toda models). Among such models we encounter the Non Abelian affine (NA) Toda models which admit electrically charged solitons [i6]. In general, the NA Toda models admit solitons with non trivial internal symmetry structure. The formulation and classification of such models with its global symmetry structure is given in terms of the decomposition of an underlying Lie algebraic structure according to a grading operator $Q$ and, in terms

\footnotetext{
${ }^{*}$ Speaker.
} 
of a pair of constant generators $\epsilon_{ \pm}$of grade \pm 1 . In particular, integrable perturbations of the WZW characterized by $\epsilon_{ \pm}$describe the dynamics of the fields parametrizing the zero grade subalgebra $\mathcal{G}_{0}$. The action manifests chiral symmetry associated to the subalgebra $\mathcal{G}_{0}^{0} \subset \mathcal{G}_{0}$ due to the fact that $Y \in \mathcal{G}_{0}^{0},\left[Y, \epsilon_{ \pm}\right]=0$. The existence of such subalgebra allows the implementation of subsidiary constraints within $\mathcal{G}_{0}^{0}$ and the reduction of the model from the group $G_{0}$ to the coset $G_{0} / G_{0}^{0}$. The structure of the coset $G_{0} / G_{0}^{0}$ viewed according to axial or vector gauging leads to different parametrizations and different actions.

We first discuss the general construction of NA Toda models and its different internal symmetry structure. Next, we discuss the structure of the coset $G_{0} / G_{0}^{0}=S L(2) \otimes$ $U(1)^{n-1} / U(1)$ according to axial and vector gaugings and explicitly construct the associated lagrangians. Subsequently, we show how the chiral symmetry of the group model becomes global under the reduction to the coset. Finally, we show that the axial and vector models are related by canonical transformation [i, $]$ preserving the Hamiltonian which also transforms topological into electric current and vice-versa.

\section{General Construction of Toda Models}

The basic ingredient in constructing Toda models is the decomposition of a Lie algebra $\mathcal{G}$ of finite or infinite dimension in terms of graded subspaces defined according to a grading operator $Q$,

$$
\left[Q, \mathcal{G}_{l}\right]=l \mathcal{G}_{l}, \quad \mathcal{G}=\oplus \mathcal{G}_{l}, \quad\left[\mathcal{G}_{l}, \mathcal{G}_{k}\right] \subset \mathcal{G}_{l+k}, l, k=0, \pm 1, \cdots
$$

In particular, the zero grade subspace $\mathcal{G}_{0}$ plays an important role since it is parametrized by the Toda fields. The grading operator $Q$ induces the notion of negative and positive grade subalgebras and henceforth the decomposition of a group element in the Gauss form,

$$
g=N B M
$$

where $N=\exp \left(\mathcal{G}_{<}\right), B=\exp \left(\mathcal{G}_{0}\right)$ and $M=\exp \left(\mathcal{G}_{>}\right)$.

The action for the Toda fields is constructed from the gauged Wess-Zumino-Witten

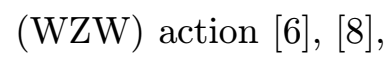

$$
\begin{aligned}
S_{G / H}(g, A, \bar{A}) & =S_{W Z W}(g) \\
& -\frac{k}{2 \pi} \int d^{2} x \operatorname{Tr}\left(A\left(\bar{\partial} g g^{-1}-\epsilon_{+}\right)+\bar{A}\left(g^{-1} \partial g-\epsilon_{-}\right)+A g \bar{A} g^{-1}\right)
\end{aligned}
$$

where $A=A_{-} \in \mathcal{G}_{<}, \bar{A}=\bar{A}_{+} \in \mathcal{G}_{>}, \epsilon_{ \pm}$are constant operators of grade \pm 1 . The action $(2,3)$ in invariant under

$$
g^{\prime}=\alpha_{-} g \alpha_{+}, \quad A^{\prime}=\alpha_{-} A \alpha_{-}^{-1}+\alpha_{-} \partial \alpha_{-}^{-1}, \quad \bar{A}^{\prime}=\alpha_{+}^{-1} \bar{A} \alpha_{+}+\bar{\partial} \alpha_{+}^{-1} \alpha_{+},
$$

where $\alpha_{-} \in \mathcal{G}_{<}, \alpha_{+} \in \mathcal{G}_{>}$. It therefore follows that $S_{G / H}(g, A, \bar{A})=S_{G / H}\left(B, A^{\prime}, \bar{A}^{\prime}\right)$.

Integrating over the auxiliary fields $A, \bar{A}$, we find the effective action,

$$
S_{e f f}(B)=S_{W Z W}(B)-\frac{k}{2 \pi} \int \operatorname{Tr}\left(\epsilon_{+} B \epsilon_{-} B^{-1}\right) d^{2} x
$$


The equations of motion are given by

$$
\bar{\partial}\left(B^{-1} \partial B\right)+\left[\epsilon_{-}, B^{-1} \epsilon_{+} B\right]=0, \quad \partial\left(\bar{\partial} B B^{-1}\right)-\left[\epsilon_{+}, B \epsilon_{-} B^{-1}\right]=0
$$

It is straightforward to derive from the eqns. of motion $\left(\overline{2}^{-6} \mathbf{6}^{1}\right)$ that chiral currents are associated to the subalgebra $\mathcal{G}_{0}^{0} \subset \mathcal{G}_{0}$ defined as $\mathcal{G}_{0}^{0}=\left\{X \in \mathcal{G}_{0}\right.$, such that $\left.\left[X, \epsilon_{ \pm}\right]=0\right\}$, i.e.,

$$
J_{X}=\operatorname{Tr}\left(X B^{-1} \partial B\right), \quad \bar{J}_{X}=\operatorname{Tr}\left(X \bar{\partial} B B^{-1}\right), \quad \bar{\partial} J_{X}=\partial \bar{J}_{X}=0
$$

In order to illustrate the different algebraic structure, consider the following examples within the affine $\mathcal{G}=\hat{S L}(n+1)$ :

1. Abelian affine Toda

$$
\begin{aligned}
Q & =(n+1) d+\sum_{l=1}^{n} \lambda_{l} \cdot H, \\
\mathcal{G}_{0} & =U(1)^{n}=\left\{h_{1}, \cdots, h_{n}\right\} \\
\epsilon_{ \pm} & =\sum_{l=1}^{n} E_{ \pm \alpha_{l}}^{(0)}+E_{\mp\left(\alpha_{1}+\cdots+\alpha_{n}\right)}^{( \pm 1)} \\
\mathcal{G}_{0}^{0} & =\emptyset
\end{aligned}
$$

2. Non Abelian affine Toda

(a)

$$
\begin{aligned}
Q & =n d+\sum_{l=2}^{n} \lambda_{l} \cdot H \\
\mathcal{G}_{0} & =S L(2) \otimes U(1)^{n-1}=\left\{E_{ \pm \alpha_{1}}, h_{1}, \cdots, h_{n}\right\} \\
\epsilon_{ \pm} & =\sum_{l=2}^{n} E_{ \pm \alpha_{l}}^{(0)}+E_{\mp\left(\alpha_{2}+\cdots+\alpha_{n}\right)}^{( \pm 1)} \\
\mathcal{G}_{0}^{0} & =U(1)=\left\{\lambda_{1} \cdot H\right\}
\end{aligned}
$$

(b)

$$
\begin{aligned}
Q & =(n-1) d+\sum_{l=2}^{n-1} \lambda_{l} \cdot H \\
\mathcal{G}_{0} & =S L(2) \otimes S L(2) \otimes U(1)^{n-2}=\left\{E_{ \pm \alpha_{1}}, E_{ \pm \alpha_{n}}, h_{1}, \cdots, h_{n}\right\} \\
\epsilon_{ \pm} & =\sum_{l=2}^{n-1} E_{ \pm \alpha_{l}}^{(0)}+E_{\mp\left(\alpha_{2}+\cdots+\alpha_{n-1}\right)}^{( \pm 1)} \\
\mathcal{G}_{0}^{0} & =U(1) \otimes U(1)=\left\{\lambda_{1} \cdot H, \lambda_{n} \cdot H\right\}
\end{aligned}
$$


(c)

$$
\begin{aligned}
Q & =(n-1) d+\sum_{l=3}^{n} \lambda_{l} \cdot H \\
\mathcal{G}_{0} & =S L(3) \otimes U(1)^{n-2}=\left\{E_{ \pm \alpha_{1}}, E_{ \pm \alpha_{2}}, E_{ \pm\left(\alpha_{1}+\alpha_{2}\right)}, h_{1}, \cdots, h_{n}\right\} \\
\epsilon_{ \pm} & =\sum_{l=3}^{n} E_{ \pm \alpha_{l}}^{(0)}+E_{\mp\left(\alpha_{3}+\cdots+\alpha_{n}\right)}^{( \pm 1)} \\
\mathcal{G}_{0}^{0} & =S L(2) \otimes U(1)=\left\{E_{ \pm \alpha_{1}}, \lambda_{1} \cdot H, \lambda_{n} \cdot H\right\}
\end{aligned}
$$

For the cases where $\mathcal{G}_{0}^{0} \neq 0$, we may impose consistently the additional constraints $J_{X}=$ $\bar{J}_{X}=0, X \in \mathcal{G}_{0}^{0}$. The construction of the gauged WZW action taking into account the subsidiary constraints reduces the model from the group $G_{0}$ to the coset $G_{0} / G_{0}^{0}$ and is given by

$$
\begin{aligned}
S_{G_{0} / G_{0}^{0}}\left(B, A_{0}, \bar{A}_{0}\right) & =S_{W Z W}(B)-\frac{k}{2 \pi} \int \operatorname{Tr}\left(\epsilon_{+} B \epsilon_{-} B^{-1}\right) d^{2} x \\
& -\frac{k}{2 \pi} \int \operatorname{Tr}\left( \pm A_{0} \bar{\partial} B B^{-1}+\bar{A}_{0} B^{-1} \partial B \pm A_{0} B \bar{A}_{0} B^{-1}+A_{0} \bar{A}_{0}\right) d^{2} x
\end{aligned}
$$

where the \pm signs correspond to axial or vector gaugings respectively. The action (2.12i) is invariant under

$$
B^{\prime}=\alpha_{0} B \alpha_{0}^{\prime}, \quad A_{0}^{\prime}=A_{0}-\alpha_{0}^{-1} \partial \alpha_{0}, \quad \bar{A}_{0}^{\prime}=\bar{A}_{0}-\bar{\partial} \alpha_{0}^{\prime}\left(\alpha_{0}^{\prime}\right)^{-1}
$$

and $\alpha_{0}^{\prime}=\alpha_{0}(z, \bar{z}) \in \mathcal{G}_{0}^{0}$ for axial and $\alpha_{0}^{\prime}=\alpha_{0}^{-1}(z, \bar{z}) \in \mathcal{G}_{0}^{0}$ for vector cases, i.e.,

$$
S_{G_{0} / G_{0}^{0}}\left(B, A_{0}, \bar{A}_{0}\right)=S_{G_{0} / G_{0}^{0}}\left(\alpha_{0} B \alpha_{0}^{\prime}=g_{0}^{f}, A_{0}^{\prime}, \bar{A}_{0}^{\prime}\right)
$$

\section{The structure of the coset $G_{0} / G_{0}^{0}$}

In this section we discuss the structure of the coset $G_{0} / G_{0}^{0}$ constructed according to axial and vector gaugings. We shall be considering the NA Toda models of case $(2 a)$ where $\mathcal{G}_{0}^{0}=U(1)$. The group element of the zero grade subgroup $G_{0}$ is parametrized as

$$
B=e^{\tilde{\chi} E_{-\alpha_{1}}} e^{R \lambda_{1} \cdot H+\sum_{l=2}^{n} \varphi_{l} h_{l}} e^{\tilde{\psi} E_{\alpha_{1}}}
$$

According to the axial gauging we can write $B$ as an element of the the zero grade subgroup $G_{0}$ is parametrized as

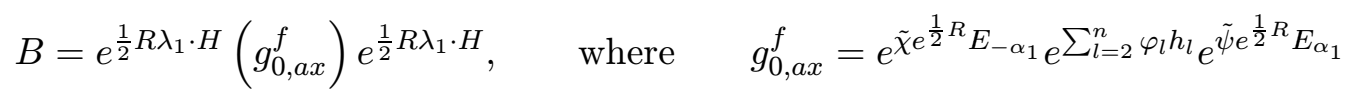

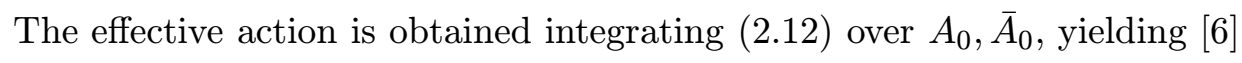

$$
\mathcal{L}_{\text {eff }}^{a x}=\frac{1}{2} \sum_{a, b=2}^{n} \eta_{a b} \partial \varphi_{a} \bar{\partial} \varphi_{b}+\frac{1}{2} \frac{\bar{\partial} \psi \partial \chi}{\Delta} e^{-\varphi_{2}}-V_{a x}, \quad \Delta=1+\frac{n+1}{2 n} \psi \chi e^{-\varphi_{2}}
$$


where $\psi=\tilde{\psi} e^{\frac{1}{2} R}, \chi=\tilde{\chi} e^{\frac{1}{2} R}$, and $V_{a x}=\sum_{l=2}^{n} e^{2 \varphi_{l}-\varphi_{l-1}-\varphi_{l+1}}+e^{\varphi_{2}+\varphi_{n}}\left(1+\psi \chi e^{-\varphi_{2}}\right)$.

The vector gauging can be implemented from the zero grade subgroup $G_{0}$ written as

$$
B=e^{u \lambda_{1} \cdot H}\left(g_{0, v e c}^{f}\right) e^{-u \lambda_{1} \cdot H}, \quad \text { where } \quad g_{0, v e c}^{f}=e^{\tilde{\chi} e^{u} E_{-\alpha_{1}}} e^{\sum_{l=1}^{n} \phi_{l} h_{l}} e^{\tilde{\psi} e^{-u} E_{\alpha_{1}}}
$$

Since $u$ is arbitrary, we may choose $u=\frac{1}{2} \ln \left(-\frac{\tilde{\psi}}{\tilde{\chi}}\right)$ so that

$$
g_{0, v e c}^{f}=e^{-t E_{-\alpha_{1}}} e^{\sum_{l=1}^{n} \phi_{l} h_{l}} e^{t E_{\alpha_{1}}}, \quad t^{2}=-\tilde{\psi} \tilde{\chi}
$$

The effective action for the vector model is [i]

$$
\begin{aligned}
\mathcal{L}_{e f f}^{v e c} & =\frac{1}{2} \sum_{a, b=1}^{n} \eta_{a b} \partial \phi_{a} \bar{\partial} \phi_{b}+\frac{\partial \phi_{1} \bar{\partial} \phi_{1}}{t^{2}} e^{-2 \phi_{1}+\phi_{2}} \\
& +\partial \phi_{1} \bar{\partial} \ln (t)+\bar{\partial} \phi_{1} \partial \ln (t)-V_{v e c} .
\end{aligned}
$$

In terms of a more convenient set of variables,

$$
A=e^{\phi_{1}}=e^{\frac{n}{n+1} R}, \quad B=A^{-1}\left(1-t^{2} e^{2 \phi_{1}-\phi_{2}}\right), \quad C_{i}=e^{\phi_{i+2}-\phi_{i+1}-\frac{R}{n}}
$$

we get

$$
\begin{aligned}
\mathcal{L}_{\text {eff }}^{\text {vec }} & =\frac{1}{2} \sum_{l=1}^{n-1}\left(\partial \ln \left(C_{i}\right) \bar{\partial} \ln \left(C_{i} C_{i+1} \cdots C_{n-1}\right)+\bar{\partial} \ln \left(C_{i}\right) \partial \ln \left(C_{i} C_{i+1} \cdots C_{n-1}\right)\right) \\
& -\frac{1}{2} \frac{\partial A \bar{\partial} B+\bar{\partial} A \partial B}{1-A B}-V_{\text {vec }}
\end{aligned}
$$

where $V_{v e c}=A C_{1}^{2} C_{2} \cdots C_{n-1}+\frac{C_{2}}{C_{1}}+\frac{C_{3}}{C_{2}}+\cdots \frac{C_{n-2}}{C_{n-1}}+\frac{B}{C_{1} C_{2} \cdots C_{n-1}^{2}}$.

\section{Chiral and Global Symmetries, from $G_{0}$ to $G_{0} / G_{0}^{0}$}

Consider the unconstrained model defined in the group $G_{0}$ with action

$$
S_{e f f}(B)=S_{W Z W}(B)-\frac{k}{2 \pi} \int \operatorname{Tr}\left(\epsilon_{+} B \epsilon_{-} B^{-1}\right) d^{2} x
$$

The action (4.1) invariant under chiral transformation

$$
B^{\prime}(\bar{z}, z)=e^{\bar{w}(\bar{z}) \lambda_{1} \cdot H} B(\bar{z}, z) e^{w(z) \lambda_{1} \cdot H},
$$

which in components reads

$$
\tilde{\chi}^{\prime}=\tilde{\chi} e^{\bar{w}(\bar{z})}, \quad \tilde{\psi}^{\prime}=\tilde{\psi} e^{w(z)}, \quad R^{\prime}=R+\bar{w}+w, \quad \varphi_{l}^{\prime}=\varphi_{l}
$$

and leads to the Noether currents (chiral)

$$
\begin{aligned}
& J_{\lambda_{1} \cdot H}=\operatorname{Tr}\left(\lambda_{1} \cdot H B^{-1} \partial B\right)=\partial R-\frac{n+1}{n} \tilde{\psi} \partial \tilde{\chi} e^{R-\varphi_{2}}, \quad \bar{J}_{\lambda_{1} \cdot H}=0 \\
& \bar{J}_{\lambda_{1} \cdot H}=\operatorname{Tr}\left(\lambda_{1} \cdot H \bar{\partial} B B^{-1}\right)=\bar{\partial} R-\frac{n+1}{n} \tilde{\chi} \bar{\partial} \tilde{\psi} e^{R-\varphi_{2}}, \quad \partial \bar{J}_{\lambda_{1} \cdot H}=0
\end{aligned}
$$


In addition the model in the group (1יili $)$ has the following topological currents (non chiral)

$$
\begin{aligned}
J_{t o p, a x}^{\mu} & =\epsilon^{\mu \nu} \partial_{\nu} R, \\
J_{t o p, v e c}^{\mu} & =\epsilon^{\mu \nu} \partial_{\nu} u, \quad u=\frac{1}{2} \ln \left(\frac{\tilde{\psi}}{\tilde{\chi}}\right)
\end{aligned}
$$

When reducing the model from the group $G_{0}$ to the coset $G_{0} / G_{0}^{0}$, the chiral symmetry reduces to a global symmetry. For axial factor group element $g_{0, a x}^{f}$ defined in (굴. no $\mathcal{G}_{0}^{0}$ generator (i.e., $R$ field to absorb the factor $\bar{w}+w$ ) and the symmetry of the axial model consists of transformations ( $(\overline{4} .3)$ such that $\bar{w}+w=0$. For the vector factor group element $g_{0, v e c}^{f}$ in ( $\left(\overline{3} . \bar{y}^{\prime}\right)$ the invariance of the field $t^{\prime}=t$ also implies $\bar{w}+w=0$. In both cases, the remaining symmetry is obtained for $\bar{w}=-w=$ const. corresponding to a global $U(1)$. Further, the reduction from the group $G_{0}$ to the coset $G_{0} / G_{0}^{0}$ is implemented by imposing the subsidiary constraint

$$
J_{\lambda_{1} \cdot H}=\bar{J}_{\lambda_{1} \cdot H}=0
$$

which allows the elimination of one degree of freedom. For the axial gauging we solve the subsidiary constraints ( $\left(\bar{A}_{1}^{-} \bar{\sigma}_{i}\right)$ for the nonlocal field $R$, i.e. the coset $G_{0} / G_{0}^{0}$ is implemented by imposing the subsidiary constraint

$$
\partial R=\left(\frac{n+1}{n}\right) \frac{\psi \partial \chi}{\Delta} e^{-\varphi_{2}}, \quad \bar{\partial} R=\left(\frac{n+1}{n}\right) \frac{\chi \bar{\partial} \psi}{\Delta} e^{-\varphi_{2}}
$$

where $\psi=\tilde{\psi} e^{\frac{1}{2} R}, \chi=\tilde{\chi} e^{\frac{1}{2} R}$ and $\Delta=1+\frac{n+1}{2 n} \psi \chi e^{-\varphi_{2}}$. For the vector gauging, we define $h=1+\tilde{\psi} \tilde{\chi} e^{-\varphi_{2}}$ and solve the same constraints (4.

$$
\begin{aligned}
\partial u & =-\frac{1}{2}\left(\frac{n-1}{n+1}+h\right) \frac{\partial R}{h-1}+\frac{1}{2} \frac{e^{-\varphi_{2}}}{h-1} \partial\left((h-1) e^{\varphi_{2}}\right), \\
\bar{\partial} u & =\frac{1}{2}\left(\frac{n-1}{n+1}+h\right) \frac{\bar{\partial} R}{h-1}-\frac{1}{2} \frac{e^{-\varphi_{2}}}{h-1} \bar{\partial}\left((h-1) e^{\varphi_{2}}\right)
\end{aligned}
$$

Finally let us discuss the relation among the topological and Noether charges of the $G_{0}$ and the $G_{0} / G_{0}^{0}$. Consider the topological charges defined from (4.4.5i)

$$
\begin{gathered}
Q_{\text {top,ax }}^{G_{0}}=\int_{-\infty}^{\infty} d x \partial_{x} R=\int_{-\infty}^{\infty} d x\left(\frac{\psi \partial \chi-\chi \bar{\partial} \psi}{\Delta}\right)=Q_{\text {Noether }, a x}^{G_{0} / G_{0}^{0}}, \\
Q_{\text {top }, \text { vec }}^{G_{0}}=\int_{-\infty}^{\infty} d x \partial_{x} u=\int_{-\infty}^{\infty} d x\left(-\left(\frac{n-1}{n+1}+h\right) \frac{\partial_{t} R}{h-1}+\frac{e^{-\varphi_{2}}}{h-1} \partial_{t}\left((h-1) e^{\varphi_{2}}\right)\right) \\
=Q_{\text {Noether,vec }}^{G_{0} / G_{0}^{0}}
\end{gathered}
$$

by virtue of eqns. (4.4. with the Noether charges associated to the global transformations $\delta \psi=\alpha \psi, \delta \chi=-\alpha \chi$ and $\delta \phi_{1}=\frac{(n+1)}{n} \delta R=\alpha, \alpha=$ const. respectively. 


\section{Axial-Vector Duality}

In this section we shall prove that the axial and vector models are related by a canonical transformation. Consider the $S L(3)$ vector model

$$
\mathcal{L}_{v e c}=\partial \ln C \bar{\partial} \ln C-\frac{1}{2} \frac{\partial A \bar{\partial} B+\bar{\partial} A \partial B}{1-A B}-A C^{2}+\frac{B}{C^{2}}
$$

In terms of the new set of more convenient variables

$$
a=A B, \quad C=d A^{-\frac{1}{2}}, \quad d=e^{\frac{1}{2} f}, \quad \theta=\ln A
$$

the lagrangian $(\bar{i} \cdot \overline{1})$ becomes

$$
\mathcal{L}_{v e c}=\left(\frac{1+3 a}{1-a}\right) \partial \theta \bar{\partial} \theta-\frac{1}{4} \partial \theta\left(\bar{\partial} f+\frac{2 \bar{\partial} a}{1-a}\right)-\frac{1}{4} \bar{\partial} \theta\left(\partial f+\frac{2 \partial a}{1-a}\right)+\frac{1}{4} \partial f \bar{\partial} f-V_{v e c}
$$

where $V_{v e c}=d^{2}+\frac{a}{d^{2}}$. The canonical momenta are given by

$$
\begin{aligned}
& \Pi_{\theta}=\frac{\delta \mathcal{L}}{\delta \dot{\theta}}=\frac{1}{2} \frac{(1+3 a)}{1-a} \dot{\theta}-\frac{1}{2}\left(\dot{f}+\frac{2 \dot{a}}{1-a}\right) \\
& \Pi_{f}=\frac{\delta \mathcal{L}}{\delta \dot{f}}=-\frac{1}{2}(\dot{\theta}+\dot{f}) \\
& \Pi_{a}=\frac{\delta \mathcal{L}}{\delta \dot{a}}=-\frac{\dot{\theta}}{1-a}
\end{aligned}
$$

and the hamiltonian is

$$
\begin{aligned}
\mathcal{H}_{v e c} & =-(1-a) \Pi_{a} \Pi_{\theta}+\Pi_{f}^{2}-(1-a) \Pi_{a} \Pi_{f}-a(1-a) \Pi_{a}^{2} \\
& +\frac{1}{4} \frac{(1+3 a)}{1-a} \theta^{\prime 2}-\frac{1}{2}\left(f^{\prime}+\frac{2 a^{\prime}}{1-a}\right) \theta^{\prime}-\frac{1}{4} f^{\prime 2}+V_{v e c}
\end{aligned}
$$

Consider the following modified lagrangian

$$
\mathcal{L}_{\text {mod }}=\mathcal{L}_{\text {vec }}-\tilde{\theta}(\partial \bar{P}-\bar{\partial} P)
$$

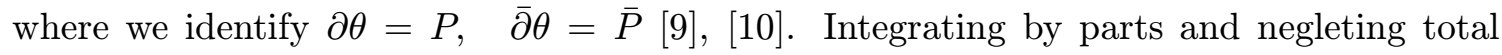
derivatives,

$$
\begin{aligned}
\mathcal{L}_{\text {mod }} & =\left(\frac{1+3 a}{1-a}\right) P \bar{P}-\frac{1}{4} P\left(\bar{\partial} f+\frac{2 \bar{\partial} a}{1-a}+\bar{\partial} \tilde{\theta}\right) \\
& -\frac{1}{4} \bar{P}\left(\partial f+\frac{2 \partial a}{1-a}-\partial \tilde{\theta}\right)+\frac{1}{4} \partial f \bar{\partial} f-V_{\text {vec }}
\end{aligned}
$$

Integrating over the auxiliary fields $P$ and $\bar{P}$ we find the effective action

$$
\mathcal{L}_{e f f}=\frac{1}{4} \partial f \bar{\partial} f-\frac{1}{4} \frac{(1-a)}{1+3 a}\left(\bar{\partial} f+\frac{2 \bar{\partial} a}{1-a}+\bar{\partial} \tilde{\theta}\right)\left(\partial f+\frac{2 \partial a}{1-a}-\partial \tilde{\theta}\right)-V
$$


and the canonical momenta are

$$
\begin{aligned}
& \Pi_{f}=\frac{\delta \mathcal{L}_{e f f}}{\delta \dot{f}}=\frac{2 a \dot{f}}{1+3 a}-\frac{\dot{a}}{1+3 a}+\frac{1}{2} \frac{(1-a)}{1+3 a} \theta^{\prime} \\
& \Pi_{\tilde{\theta}}=\frac{\delta \mathcal{L}_{e f f}}{\delta \dot{\tilde{\theta}}}=\frac{1}{2} \frac{(1-a)}{1+3 a}\left(\dot{\tilde{\theta}}-f^{\prime}\right)-\frac{g^{\prime}}{1+3 a} \\
& \Pi_{a}=\frac{\delta \mathcal{L}_{e f f}}{\delta \dot{a}}=\frac{\left(\tilde{\theta}^{\prime}-\dot{f}\right)}{1+3 a}-\frac{2 a}{(1-a)(1+3 a)}
\end{aligned}
$$

The hamiltonian is

$$
\begin{aligned}
\mathcal{H}_{\text {mod }} & =-\frac{1}{2}(1-a) \Pi_{a} \tilde{\theta}^{\prime}+\Pi_{f}^{2}-(1-a) \Pi_{a} \Pi_{f}-a(1-a) \Pi_{a}^{2} \\
& +\frac{(1+3 a)}{1-a} \Pi_{\tilde{\theta}}^{2}-\frac{1}{2}\left(f^{\prime}+\frac{2 a^{\prime}}{1-a}\right) \Pi_{\tilde{\theta}}-\frac{1}{4}{f^{\prime}}^{2}+V_{v e c}
\end{aligned}
$$

The canonical transformation

$$
\Pi_{\theta}=-\frac{1}{2} \tilde{\theta}^{\prime}, \quad \theta^{\prime}=-2 \Pi_{\tilde{\theta}}
$$

preserves the Poisson bracket structure and provide the equality of the hamiltanians $\mathcal{H}_{\text {mod }}=$ $\mathcal{H}_{\text {vec }}$. If we now substitute

$$
\tilde{\theta}=2 \ln \left(\frac{\psi}{\chi}\right), \quad a=1+\psi \chi e^{-\varphi_{2}}, \quad f=-\varphi_{2}
$$

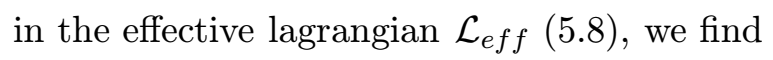

$$
\mathcal{L}_{e f f}=\frac{1}{2} \partial \varphi_{2} \bar{\partial} \varphi_{2}+\frac{\partial \chi \bar{\partial} \psi}{\Delta} e^{-\varphi_{2}}-V
$$

which is precisely the axial lagrangian for $\mathcal{G}_{0}=S L(3)$. Therefore the axial and the vector models are related by the canonical transformation $(\overline{5}, 1 \overline{1} 1)$ preserving the hamiltonians. In fact, $\theta$ and $\tilde{\theta}$ are isometric variables generating Noether charges $(4 . \overline{9})$ and $(4 . \overline{1} 0)$ under global transformations.

\section{Concluding Remarks}

We have seen that the crucial ingredient which allows the construction of the axial and vector models is the existence of non trivial subgroup $G_{0}^{0}$ which in the example developed here, $G_{0}^{0}=U(1)$. The same strategy works equally well for generalized multicharged NA Toda models such as those constructed in ref. [1]

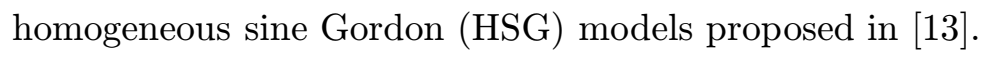

The question of constructing integrable models with non abelian internal symmetry arises naturally. For instance, in the case of example $(2 c)$ of section 2 (eqn. (2.11)), the group model $(2.4)$ admits invariance under chiral $\mathcal{G}_{0}^{0}=S L(2) \otimes U(1)$ transformations and is the natural prototype to describe solitons carrying nonabelian degrees of freedom.

An interesting and intriguing subclass of NA Toda models correspond to the following three affine Kac-Moody algebras, $B_{n}^{(1)}, A_{2 n}^{(2)}$ and $D_{n+1}^{(2)}$. Their axial and vector actions were 
constructed in $[\overline{\bar{i}}]$ and shown to be identical. In fact, those affine algebras satisfy the no torsion condition proposed in [i, $]$ which is fulfilled by Lie algebras possesseing $B_{n}$-tail like Dynkin diagrams (see for instance appendix $\mathrm{N}$ of [i] $\left.\mathbf{i}_{i}\right]$ ). The very same selfdual models were shown to possess an exact S-matrix coinciding with certain Thirring models coupled to affine abelian Toda models in ref. [i]

Acknowledgments We thank O. Babelon, J.L. Miramontes and J. Sanchez-Guillen for discussions. We are grateful to CAPES, CNPq, FAPESP and UNESP for financial support.

\section{References}

[1] L.D. Faddeev and L. Takhtajan, "Hamiltonian methods in the Theory of Solitons", (Springer, Berlin ),1987

[2] A. N. Leznov, M. V. Saveliev, Group Theoretical Methods for Integration of Nonlinear Dynamical Systems, Progress in Physics, Vol. 15 (1992), Birkhauser Verlag, Berlin

[3] J. Balog, L. Feher, L. O'Raifeartaigh, P. Forgas and A. Wipf, Ann. of Phys. 203 (1990) 76

[4] H. Aratyn, L.A. Ferreira, J.F. Gomes and A.H. Zimerman, Phys. Lett B 254 (1991) 372

[5] L.A. Ferreira, J.F. Gomes, A. Schwimmer and A.H. Zimerman, Phys. Lett. 274B (1992) 65

[6] J.F. Gomes, E.P. Gueuvoghlanian, G.M. Sotkov and A.H. Zimerman, Nucl. Phys. B598 (2001) 615, hepth/0011187

[7] J.F. Gomes, E.P. Gueuvoghlanian, G.M. Sotkov and A.H. Zimerman, Ann. of Phys. 289 (2001) 232 , hepth/0007116

[8] J.F. Gomes, E.P. Gueuvoghlanian, G.M. Sotkov and A.H. Zimerman, Nucl. Phys. B606 (2001) 441 , hepth/0007169

[9] T. Busher, Phys. Lett. 159B (1985) 127,Phys. Lett. 194B (1987) 59, Phys. Lett. 201B (1988) 466

[10] E. Kiritsis, Mod. Phys. Lett. A6 (1991) 2871

[11] I. Cabrera-Carnero, J.F. Gomes, G.M. Sotkov and A.H. Zimerman, Nucl. Phys. B634 (2002) 433 , hepth $/ 0201047$

[12] J.F. Cornwell, Group Theory in Physics, Vol. 3, Academic Press (1989)

[13] C.R. Fernandez-Pousa, M.V. Gallas, T.J. Hollowood and J.L. Miramontes, Nucl. Phys. B484 (1997) 609, Nucl. Phys. B499 (1997) 673

[14] V.A. Fateev, Nucl. Phys. B479 (1996) 594 